Discrete Comput Geom 30:167-176 (2003)

DOI: $10.1007 / \mathrm{s} 00454-003-0001-\mathrm{z}$

\title{
Total Curvature and Spiralling Shortest Paths*
}

\author{
Imre Bárány, ${ }^{1}$ Krystyna Kuperberg, ${ }^{2}$ and Tudor Zamfirescu ${ }^{3}$ \\ ${ }^{1}$ Rényi Institute of Mathematics, Hungarian Academy of Sciences, \\ POB 127, 1364 Budapest, Hungary \\ barany@ renyi.hu \\ and \\ Department of Mathematics, University College London, \\ Gower Street, London WC1E6BT, England \\ ${ }^{2}$ Department of Mathematics, Auburn University, \\ Auburn, AL 36830-5310, USA \\ kuperkm@math.auburn.edu \\ ${ }^{3}$ Fachbereich Mathematik, Universität Dortmund, \\ 44221 Dortmund, Germany \\ tudor.zamfirescu@mathematik.uni-dortmund.de
}

\begin{abstract}
This paper gives a partial confirmation of a conjecture of Agarwal, Har-Peled, Sharir, and Varadarajan that the total curvature of a shortest path on the boundary of a convex polyhedron in $\mathbb{R}^{3}$ cannot be arbitrarily large. It is shown here that the conjecture holds for a class of polytopes for which the ratio of the radii of the circumscribed and inscribed ball is bounded. On the other hand, an example is constructed to show that the total curvature of a shortest path on the boundary of a convex polyhedron in $\mathbb{R}^{3}$ can exceed $2 \pi$. Another example shows that the spiralling number of a shortest path on the boundary of a convex polyhedron can be arbitrarily large.
\end{abstract}

\section{Introduction}

The total curvature of a $C^{2}$ path $C$ parameterized by arc length $s$ in $\mathbb{R}^{n}, r(s)$, is defined as $\int_{C}\left|r^{\prime \prime}(s)\right| d s$. Fenchel proved in 1929 for $\mathbb{R}^{3}$ and Borsuk in 1947 for any $\mathbb{R}^{n}$ that the total curvature of a closed curve is bounded from below by $2 \pi$, with the equality holding

* I. Bárány's research was supported in part by Hungarian National Science Foundation Grants \#T-032452 and \#T-029255. K. Kuperberg's research was supported in part by US National Science Foundation Grant \#DMS-9704558. 
only for convex simple closed curves in $\mathbb{R}^{2}$. The total curvature of a polygonal path $P=\left[z_{0}, z_{1}, \ldots, z_{n}\right]$ is defined as

$$
t(P)=\sum_{i=1}^{n-1}\left(\pi-\angle z_{i-1} z_{i} z_{i+1}\right) .
$$

Let $\mathcal{K}$ be the set of all compact convex polyhedra in $\mathbb{R}^{3}$. Let $\mathcal{T}=\{t(P)\}$, where $P$ is a shortest path joining two points on the boundary of a polyhedron $K \in \mathcal{K}$. It has been asked in [1] whether the set $\mathcal{T}$ is bounded.

We prove here, in Theorem 1 below, that the conjecture holds for polytopes $K$ for which the ratio $R / r$ is bounded from above: here $R$ and $r$, respectively, are the radii of the circumscribed and inscribed ball to $K$.

We define the spiralling number $s(P)$ for the path $P$ from $a$ to $b$ on the polytopal surface by considering a variable point $x \in P \backslash\{a, b\}$, writing this point in cylindrical coordinates as $(2 \pi \varphi(x), r(x), z(x))$ where the $z$-axis is the line through $a$ and $b$, and $\varphi$ is a continuous function. Set now

$$
s(P)=\lim _{x \rightarrow a, y \rightarrow b}|\varphi(x)-\varphi(y)| .
$$

This measures how many times the path spirals around the line through $a$ and $b$.

The proof method of Theorem 1 would work for all polytopes if the function $\varphi$ had bounded variation. This, however, will be shown to be false. In Section 4 we construct needle-like polytopes $K$ with shortest path $P$ such that $s(P)$ is arbitrarily large. Even more surprisingly, the example can be modified so that $P$ spirals around the line through $a$ and $b 100$ times in one direction, then 200 times in the opposite direction, then 1000 times in the first direction, etc.

The total curvature $t(P)$ of a planar path $P$ is bounded sharply by $2 \pi$ and this bound is the lowest possible. A triangle with one of the angles very close to $\pi$ and two points on the two sides adjacent to the wide angle but close to the vertices at the acute angles provides a simple example.

In Section 3 we construct an example of a shortest path on the boundary of a convex polyhedron in $\mathbb{R}^{3}$ for which the $2 \pi$ bound does not hold. On some level the example resembles the planar example involving a triangle with one of the angles close to $\pi$.

\section{Bounded Total Curvature of Shortest Paths for $R / r$ Bounded}

On the boundary of a polytope consider a shortest path $P$ with non-smooth points $z_{0}, z_{1}, \ldots, z_{n}$. Put $x_{i}=\left(z_{i}-z_{i-1}\right) /\left\|z_{i}-z_{i-1}\right\|(i=1, \ldots, n)$. Let $u_{i}$ be the outernormal (of the interior) of the facet of $z_{i-1}$ and $z_{i}$, and let $\xi_{i}$ be the angle between $x_{i}$ and $x_{i+1}$ $(i=1, \ldots, n-1)$. Then the total curvature of $P$ is $\sum_{i=1}^{n-1} \xi_{i}$. This can be easily checked. We remark that $x_{i}-x_{i+1}=\lambda_{i}\left(u_{i}+u_{i+1}\right)$ with $\lambda_{i}>0$.

Lemma 1. If there is a unit vector $v$ such that $u_{i} v \geq \eta>0$ for all $i$, then $\sum_{i=1}^{n-1} \xi_{i}<\pi / \eta$. 
Proof. First note that $\xi<(\pi / 2) \sin \xi$ if $\xi<\pi / 2$. Then

$$
\begin{aligned}
\sum_{i=1}^{n-1} \xi_{i} & =2 \sum_{i=1}^{n-1} \frac{\xi_{i}}{2}<2 \sum_{i=1}^{n-1} \frac{\pi}{2} \sin \frac{\xi_{i}}{2} \\
& =\frac{\pi}{2} \sum_{i=1}^{n-1}\left\|x_{i}-x_{i+1}\right\|=\frac{\pi}{2} \sum_{i=1}^{n-1}\left\|\lambda_{i}\left(u_{i}+u_{i+1}\right)\right\| .
\end{aligned}
$$

Since $\left\|u_{i}+u_{i+1}\right\| \leq 2$ and $\left(u_{i}+u_{i+1}\right) v \geq 2 \eta$, we have

$$
\left\|u_{i}+u_{i+1}\right\| \leq \frac{\left(u_{i}+u_{i+1}\right) v}{\eta} .
$$

Hence

$$
\sum_{i=1}^{n-1} \xi_{i}<\frac{\pi}{2} \sum_{i=1}^{n-1} \lambda_{i} \frac{\left(u_{i}+u_{i+1}\right) v}{\eta}=\frac{\pi}{2 \eta} \sum_{i=1}^{n-1}\left(x_{i}-x_{i+1}\right) v=\frac{\pi}{2 \eta}\left(x_{1}-x_{n}\right) v \leq \frac{\pi}{\eta} .
$$

Denote by $B$ the closed unit ball in $\mathbb{R}^{3}$.

Theorem 1. Let a polytope $Q$ satisfy $r B \subset Q \subset B$. Then the total curvature of any shortest path on the boundary of $Q$ is less than $4 \pi^{2} r^{-2}$.

Proof. Let $P$ be a shortest path between points $a$ and $b$ on the boundary bd $Q$ of $Q$. The length of $P$ is less than $\pi$. Indeed, any plane through $a$ and $b$ intersects the boundary of $B$ along a circle of length at most $2 \pi$, and the boundary of $Q$ along a polygon of even smaller length. So one of the two broken lines into which $a$ and $b$ divide the polygon must have length less than $\pi$, and $P$ cannot be longer. For a non-zero vector $v \in \mathbb{R}^{3}$ define the $v$-shadow $S(v)$ of $Q$ as

$$
S(v)=\operatorname{bd} Q \cap\left\{\frac{r}{2} B+\lambda v \mid \lambda \geq 0\right\} .
$$

Assume that $u$ is the outernormal at an interior point $f$ of a facet $F$ of $Q$ with $f \in S(v)$. We claim that $u v /\|v\|>r / 2$. Indeed, the plane $\Pi$ of $F$ does not meet the interior of $r B$. Let $\gamma$ be the angle between $u$ and $v$. Of course, $\|f\|<1$. The distance from $f$ to the line through the origin $\mathbf{0}$ and $v$ is at most $r / 2$ because $f \in S(v)$. Further, the distance from $\mathbf{0}$ to $\Pi$ is at least $r$. Therefore

$$
\frac{u v}{\|v\|}=\cos \gamma>\frac{r}{2},
$$

which proves the claim. We are going to define points $v_{1}, v_{2}, \ldots, v_{k}$ on $P$ by recursion. Set $v_{1}=a$ and assume that $v_{i}$ has been defined. Let $v_{i+1}$ be the first point on $P$, going from $v_{i}$ to $b$, which is not in the interior of $S\left(v_{i}\right)$. Write $P_{i}$ for the part of the path $P$ between $v_{i}$ and $v_{i+1}$. Since the length of $P_{i}$ is at least $\left\|v_{i}-v_{i+1}\right\| \geq r / 2$ and the length of $P$ is less than $\pi$, there are only $k<2 \pi / r$ paths $P_{i}$, and we put $v_{k+1}=b$. Note that 
Lemma 1 applies to $P_{i}$ with $\eta=r / 2$ by the above claim. Hence the total curvature of $P_{i}$ is less than $2 \pi / r$. Summing up, the total curvature of $P$ is less than

$$
k \frac{2 \pi}{r}<\frac{4 \pi^{2}}{r^{2}} .
$$

Now we formulate Theorem 1 differently. Let $E$ be the ellipsoid of largest volume included in the polytope $Q$. As the total curvature is invariant under angle preserving linear transformation, we may assume that $E$ has half-axes $a, b, c$ with $0<a \leq b \leq$ $c=1$. Call $Q$ needle-like if $b$ is small, and pancake-like if $a$ is small compared with $b$. Theorem 1 on the boundedness of total curvature holds for convex bodies that are not pancake-like. Only moderate effort is needed to prove the following: if the conjecture holds for needle-like convex bodies, then it holds for pancake-like ones as well. So it would be enough to prove the conjecture for needle-like convex bodies. This would follow if the total variation of $\varphi$, the function defined in the first paragraph, were bounded. However, this is not true: an example showing this is given in Section 4.

\section{The Total Curvature $t(P)>2 \pi$ : An Example}

We construct a convex body $\Delta$ with two points on the boundary such that the total curvature of the shortest path joining the points exceeds $2 \pi . \Delta$ is constructed in four steps, with certain unbounded convex bodies $U, X$, and $Y_{\alpha}$ described in steps 1-3. The Cartesian coordinates of a point $x \in \mathbb{R}^{n}$ are denoted by $x^{(1)}, \ldots, x^{(n)}$.

For $0<\epsilon<\sqrt{2} / 8 \pi$ and $i=1,2$, define $V_{i}$ to be the plane $\left\{\left(x^{(1)}, x^{(2)}, x^{(3)}\right) \in \mathbb{R}^{3}\right.$ | $\left.x^{(3)}=(-1)^{i} \epsilon\right\}$. Let $A_{i}$ be the parabola in $\left\{\left(x^{(1)}, x^{(2)}, x^{(3)}\right) \in V_{i} \mid x^{(2)}=\left(x^{(1)}\right)^{2}\right\}$ and let $B_{i}$ be the parabola in $\left\{\left(x^{(1)}, x^{(2)}, x^{(3)}\right) \in V_{i} \mid x^{(2)}=2\left(x^{(1)}\right)^{2}\right\}$. Denote by $v_{i}$ the common vertex of $A_{i}$ and $B_{i}$, by $a_{i}$ the focus of $A_{i}$, and by $b_{i}$ the focus of $B_{i}$. Thus $a_{i}=\left(0, \frac{1}{4},(-1)^{i} \epsilon\right), b_{i}=\left(0, \frac{1}{8},(-1)^{i} \epsilon\right)$, and $v_{i}=\left(0,0,(-1)^{i} \epsilon\right)$. Denote by $R_{i}$ the convex region in the plane $V_{i}$ bounded by $A_{i}$. Put

$$
L=\left\{\left(x^{(1)}, x^{(2)}, x^{(3)}\right) \in \mathbb{R}^{3} \mid\left(x^{(2)}-\epsilon\right)^{2}+\left(x^{(3)}\right)^{2}=2 \epsilon^{2}, x^{(1)}=0, x^{(2)} \leq 0\right\}
$$

and

$$
\Gamma=\left\{\left(x^{(1)}, x^{(2)}, x^{(3)}\right) \in \mathbb{R}^{3} \mid\left(x^{(2)}-\epsilon\right)^{2}+\left(x^{(3)}\right)^{2}=2 \epsilon^{2},-\frac{1}{2} \leq x^{(1)} \leq \frac{1}{2}, x^{(2)} \leq 0\right\} .
$$

Thus $L$ is a quarter-circle joining the vertices $v_{1}$ and $v_{2}$ and $\Gamma$ is a surface obtained by sliding $L$ along the segment $\left\{\left(x^{(1)}, x^{(2)}, x^{(3)}\right) \in \mathbb{R}^{3} \mid-\frac{1}{2} \leq x^{(1)} \leq \frac{1}{2}, x^{(2)}=x^{(3)}=0\right\}$. Note that $|L|=\pi \epsilon / \sqrt{2}<\frac{1}{8}$. Let

$$
U=\operatorname{conv}\left(A_{1} \cup A_{2} \cup \Gamma\right)
$$

be the convex hull of the union $A_{1} \cup A_{2} \cup \Gamma$.

Suppose that $G$ is a line in $V_{2}$, crossing $A_{2}$, and parallel to the directrix of $A_{2}$. Denote by $\delta_{B}$ the distance between $G$ and the directrix of $B_{2}$, and by $\delta_{A}$ the distance between $G$ and the directrix of $A_{2}$. Note that if a shortest path $P$ in bd $U$ joining $G$ with $b_{1}$ passes through $v_{1}$ and $v_{2}$, then the length $|P|$ of $P$ equals $\delta_{B}+|L|$. 


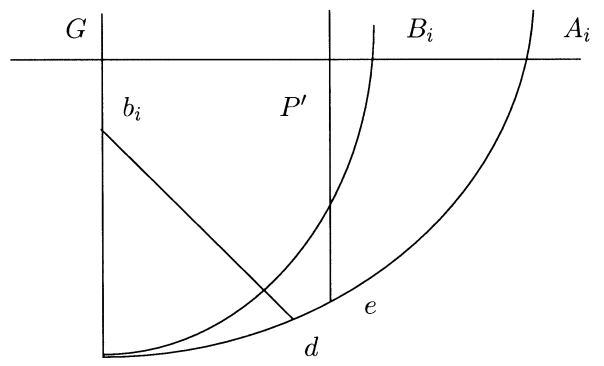

Fig. 1. The parabolas.

Lemma 2. The shortest path $P$ on the boundary of $U$ joining $G$ with $b_{1}$ is unique and passes through the vertices $v_{1}$ and $v_{2}$.

Proof. Suppose that $P^{\prime}$ is a shortest path in bd $U$ joining $b_{1}$ with $G$, different from $P$, crossing $A_{1}$ at $d=\left(d^{(1)}, d^{(2)}, d^{(3)}\right)$ and $A_{2}$ at $e=\left(e^{(1)}, e^{(2)}, e^{(3)}\right)$, see Fig. 1 . Note that $d^{(2)}<e^{(2)}$.

To show that $\left|P^{\prime}\right|>|P|$, consider the two cases: $\left|e^{(1)}\right| \geq \frac{1}{2}$ and $\left|e^{(1)}\right|<\frac{1}{2}$. We have

1. If $\left|e^{(1)}\right| \geq \frac{1}{2}$, then $e^{(2)} \geq \frac{1}{4}$ and $\left|P^{\prime}\right|>\operatorname{dist}(G, e)+\operatorname{dist}\left(e, a_{2}\right)=\delta_{A}=\delta_{B}+\frac{1}{8}>$ $\delta_{B}+|L|=|P|$.

2. If $\left|e^{(1)}\right|<\frac{1}{2}$, then $P^{\prime}$ projects onto $P$ decreasing its length.

Remark 1. If $P^{\prime}(e)$ denotes the shortest path in bd $U$ joining $b_{1}$ with $G$ and passing through $e \in A_{2}$, then the length $\left|P^{\prime}(e)\right|$ is a monotone function of $e^{(1)}$ for $0<e^{(1)}<\frac{1}{2}$ (and for $-\frac{1}{2}<e^{(1)}<0$ ).

In the next step of the construction, we modify $U$ to obtain a convex unbounded slab $X$. Let $L^{\prime}$ be a convex curve in the $\left(x^{(1)}, x^{(2)}\right)$-plane close to the segment $\left(x^{(1)}, 0,0\right)$, $-\frac{1}{2} \leq x^{(1)} \leq \frac{1}{2}$, and let $\Gamma^{\prime}$ be a positively curved surface in $U$, close to $\Gamma$, obtained by sliding $L$ along $L^{\prime}$, keeping $L$ parallel to the $\left(x^{(2)}, x^{(3)}\right)$-plane, and the midpoint of $L$ and $L^{\prime}$. Denote by $X$ the convex hull $\operatorname{conv}\left(A_{1} \cup A_{2} \cup \Gamma^{\prime}\right)$. We require of $L^{\prime}$ (and consequently of $\left.\Gamma^{\prime}\right)$ that

1. $\Gamma^{\prime}$ contains the quarter-circle $L$;

2. the path $P$ is the shortest path joining $G$ with $b_{1}$ on the boundary of $X$.

By Remark 1, such an $X$ exists. Note that $X$ is a thin convex unbounded slab whose top and bottom are (horizontal) planar regions $R_{i}^{\prime}$ 's containing $R_{i}$ 's and with a positively curved side surface close to the vertices $v_{1}$ and $v_{2}$, see Fig. 2 .

For $0<\alpha<\pi / 4$, let $Y_{\alpha}$ be a "slant slab" obtained from $X$ by " $\alpha$-slanting" the plane $V_{1}$ at the vertex $v_{1}$ in the direction perpendicular to the $x^{(1)}$-axis as follows: Let $\Omega_{\alpha}(x)=\left(x^{(1)}, x^{(2)} \cos \alpha,-\epsilon-x^{(2)} \sin \alpha\right)$ and let $Y_{\alpha}=\operatorname{conv}\left(X \cup \Omega_{\alpha}\left(R_{1}^{\prime}\right)\right)$. Note that the portion of $Y_{\alpha}$ above the $\left(x^{(1)}, x^{(2)}\right)$-plane is identical to that of $X$. 


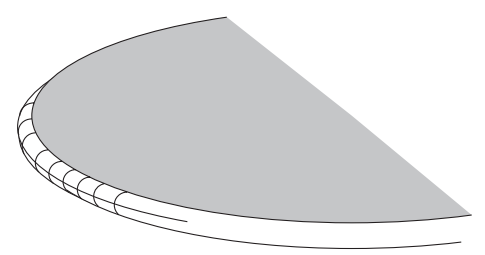

Fig. 2. The unbounded slab $X$

Lemma 3. The shortest path $\tilde{P}$ joining $G$ with $b_{1}^{\prime}=\Omega_{\alpha}\left(b_{1}\right)$ on the boundary of $Y_{\alpha}$ passes through the vertices $v_{1}$ and $v_{2}$.

Proof. A path $P^{\prime}$ joining $G$ with $b_{1}^{\prime}$ in bd $Y_{\alpha}$ can be mapped (keeping the first coordinate and the distance to the line $(t, 0,-\epsilon)$ unchanged) onto the boundary of $X$ to a path joining $G$ with $b_{1}$. Such a projection does not increase the length of the path. Hence, if $P^{\prime} \neq \tilde{P}$, $\left|P^{\prime}\right|>\delta_{B}+\pi \epsilon=|P|=|\tilde{P}|$.

Remark 2. For $\tilde{P}$ as defined in Lemma 3, the total curvature of $\tilde{P}, t(\tilde{P})$, equals $\pi-\alpha$.

Denote by $S$ the side boundary of $Y_{\alpha}$, i.e., $S=$ bd $Y_{\alpha} \backslash\left(R_{2}^{\prime} \cup \Omega_{\alpha}\left(R_{1}^{\prime}\right)\right)$. For a point $w$, $\operatorname{conv}\left(Y_{\alpha}, w\right)$ denotes the convex hull of $Y_{\alpha}$ and $w$. For a point $w \notin Y_{\alpha}$, define an attached cone with vertex $w, \operatorname{con}(w)$, as the closure of $\operatorname{conv}\left(Y_{\alpha}, w\right) \backslash Y_{\alpha}$ provided that it does not intersect $R_{2}^{\prime} \cup \Omega_{\alpha}\left(R_{1}^{\prime}\right)$.

Denote by $P_{0}$ the shortest path in $S$, a quarter-circle, joining $v_{2}$ with $v_{3}=(0,-\epsilon, 0)$. Let $w_{1}, w_{2}$, and $w_{3}$ be points close to $P_{0}$ for which attached cones con $\left(w_{i}\right)$ are defined and are pairwise disjoint. Note that $\tilde{Y}=Y_{\alpha} \cup \operatorname{con}\left(w_{1}\right) \cup \operatorname{con}\left(w_{2}\right) \cup \operatorname{con}\left(w_{3}\right)$ is convex.

First choose points $w_{1}, w_{2}$, and $w_{3}$ so that

1. each $\operatorname{con}\left(w_{i}\right), i=1,2,3$, intersects $P_{0}$ at one point $t_{i}$ with $\operatorname{dist}\left(t_{1}, t_{2}\right)=\operatorname{dist}\left(t_{2}, t_{3}\right)$;

2. $\operatorname{con}\left(w_{1}\right)$ and $\operatorname{con}\left(w_{3}\right)$ are on the same side $P_{0}$, and $\operatorname{con}\left(w_{2}\right)$ is on the other side of $P_{0}$.

Then move the points $w_{1}, w_{2}$, and $w_{3}$ slightly towards $P_{0}$ to obtain points $w_{1}^{\prime}, w_{2}^{\prime}$, and $w_{3}^{\prime}$ such that the attached cones $C_{i}=\operatorname{con}\left(w_{i}^{\prime}\right)$ intersect $P_{0}$ and the shortest path $K$ joining $G$ and $b_{1}^{\prime}$ in bd $\tilde{Y}$ crosses each $C_{i}$, but passes through $v_{1}$, $v_{2}$, and $v_{3}$, see Fig. 3.

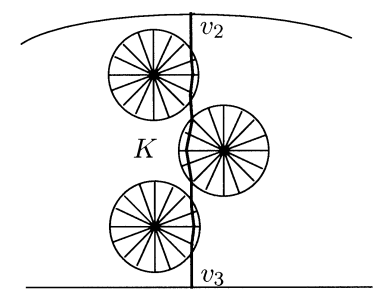

Fig. 3. Attached cones. 


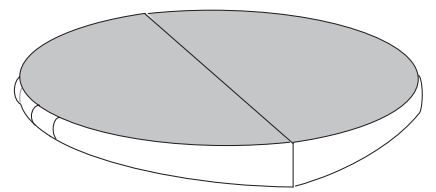

Fig. 4. The double slab $\Delta$.

Note that $K$ is not planar and as follows from the work of Fenchel [3] (alternatively, see [2] or [4]) its total curvature is greater than that of $P_{0}$. We have

Lemma 4. The total curvature $t(K)=\pi+\beta-\alpha$, where $\beta>0$.

Finally, take $\alpha<\beta$. The line $G$ consists of points $(t, g, \epsilon)$, where $-\infty<t<\infty$. Let $\Delta_{1}$ be the part of $\tilde{Y}$ cut off by the plane $x^{(2)}=g$. Let $\Delta_{2}$ be a symmetrical copy of $\Delta_{1}$ and $\Delta=\Delta_{1} \cup \Delta_{2}$ with $\Delta_{1}$ and $\Delta_{2}$ glued along the side $x^{(2)}=g$ (see Fig. 4). Let $K^{\prime \prime}$ and $b_{1}^{\prime \prime}$ be the path and the point in $\Delta_{2}$ corresponding to $K^{\prime}$ and $b_{1}^{\prime}$. For sufficiently large $g$, the path $\bar{K}=K^{\prime} \cup K^{\prime \prime}$ is the shortest path joining $b_{1}^{\prime}$ and $b_{1}^{\prime \prime}$ in $\Delta$. We have $T(\bar{K})=2(\pi-\beta+\alpha)>2 \pi$. Clearly, $\Delta$ is convex.

A polyhedral example can be obtained by a suitable approximation of $\Delta$.

Theorem 2. There exist a convex polyhedron $M \subset \mathbb{R}^{3}$ with two points $x$ and $y$ on the boundary of $M$ such that the total curvature of the (unique) shortest path joining $x$ and $y$ exceeds $2 \pi$.

\section{Spiralling Shortest Paths}

Here we construct polytopal surfaces possessing shortest paths of arbitrarily large spiralling number. The intrinsic metric on these surfaces will be denoted by $\delta$.

Theorem 3. Let $n$ be an integer. There exist a convex polytope $Q$ and a shortest path $P$ between two points on the boundary of $Q$ with $s(P) \geq n$.

Proof. Our example is the boundary of the convex hull of a family of equilateral triangles, each two of which have pairwise parallel edges. Suppose the construction performed up to the equilateral triangle $a b c$, so that, from a fixed point $x_{0}$ of the (already constructed) surface, the intrinsic distance $\delta\left(x_{0}, b c\right)$ to $b c$ (which is $\min _{y \in b c} \delta\left(x_{0}, y\right)$ ) is smaller than the distance to any other side of $a b c$. Let $\varepsilon$ be the smaller of the two differences. We now construct the next triangle $a^{\prime} b^{\prime} c^{\prime}$. We do so that

$$
\delta\left(x_{0}, a^{\prime} b^{\prime}\right)<\min \left\{\delta\left(x_{0}, b^{\prime} c^{\prime}\right), \delta\left(x_{0}, c^{\prime} a^{\prime}\right)\right\}
$$

and any shortest path from $x_{0}$ to any point $w$ of $a^{\prime} b^{\prime}$ necessarily crosses $b c$. Let $z^{\prime}$ be such that $\operatorname{conv}\left\{z^{\prime}, a, b, c\right\}$ is a regular pyramid containing the already constructed surface. 


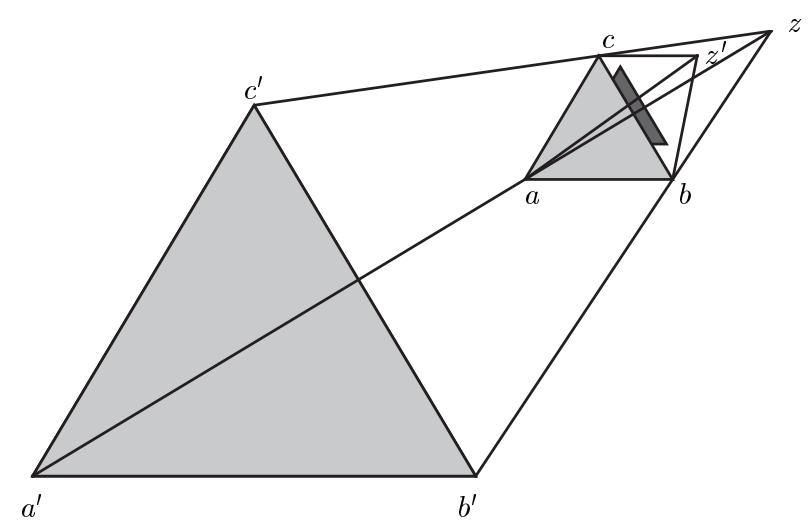

Fig. 5. Stacking triangles.

Then we can find a point $z$ behind $z^{\prime}$ (see Fig. 5) but close to the pyramid axis such that

$$
\|z-a\|=\|z-b\|<\|z-c\|
$$

and $\operatorname{conv}\{z, a, b, c\}$ includes $\operatorname{conv}\left\{z^{\prime}, a, b, c\right\}$. We shall choose $q>3$ and put $a^{\prime}=$ $z+q(a-z), b^{\prime}=z+q(b-z)$ and $c^{\prime}=z+q(c-z)$. The angles $\alpha=\angle a b b^{\prime}$ and $\gamma=\angle c b b^{\prime}$ satisfy

$$
\pi / 2<\gamma<\alpha
$$

if $z$ is close enough to the pyramid axis.

Consider an unfolding of the surface on the plane of $a, b, a^{\prime}, b^{\prime}$ without cutting along $b b^{\prime}$, but cutting along $a a^{\prime}$, and keep the notation. Let $s$ be the orthogonal projection of $c$ on the line $L$ through $a$ and $b$. If $\alpha+\gamma-\pi$ is small enough, which is the case if $z$ is far enough, then $\delta(c, s)$ is as small as desired, in particular smaller than $\varepsilon / 2$. Let $v \in a b, s^{\prime} \in b s$, see Fig. 6 .

It is easily verified that $\delta\left(s^{\prime}, w\right)-\delta(v, w)$ increases when $w$ moves from $b^{\prime}$ to $a^{\prime}$. Thus

$$
\delta\left(s^{\prime}, w\right)-\delta(v, w) \leq \delta\left(s^{\prime}, a^{\prime}\right)-\delta\left(v, a^{\prime}\right) .
$$

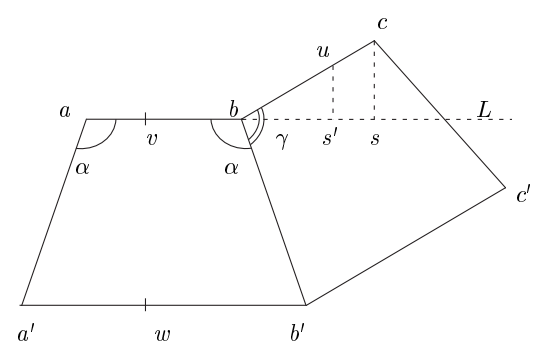

Fig. 6. Unfolding surface. 
If $\alpha+\gamma-\pi$ is small enough and $q$ large enough, then

$$
\delta\left(s, a^{\prime}\right)-\delta\left(a, a^{\prime}\right)<\frac{\varepsilon}{2} .
$$

Since $\delta\left(v, a^{\prime}\right) \geq \delta\left(a, a^{\prime}\right)$ and $\delta\left(s^{\prime}, a^{\prime}\right) \leq \delta\left(s, a^{\prime}\right)$,

$$
\delta\left(s^{\prime}, a^{\prime}\right)-\delta\left(v, a^{\prime}\right)<\frac{\varepsilon}{2} .
$$

Hence

$$
\delta\left(s^{\prime}, w\right)<\delta(v, w)+\frac{\varepsilon}{2} .
$$

This ensures that any shortest path from $x_{0}$ to some point $w \in a^{\prime} b^{\prime}$ crosses $b c$. Indeed, if the above path crosses $a b$ at $v$, then

$$
\begin{aligned}
\delta\left(x_{0}, v\right)+\delta(v, w) & \geq \delta\left(x_{0}, a b\right)+\delta(v, w) \\
& \geq \delta\left(x_{0}, b c\right)+\varepsilon+\delta(v, w)=\delta\left(x_{0}, u\right)+\frac{\varepsilon}{2}+\frac{\varepsilon}{2}+\delta(v, w) \\
& >\delta\left(x_{0}, u\right)+\delta(c, s)+\delta\left(s^{\prime}, w\right) \geq \delta\left(x_{0}, u\right)+\delta\left(u, s^{\prime}\right)+\delta\left(s^{\prime}, w\right),
\end{aligned}
$$

$u$ being a point of $b c$ closest to $x_{0}$, and $s^{\prime}$ the orthogonal projection of $u$ on $L$; we got a contradiction. If the path crosses $c a$ at $v^{\prime}$, say, then

$$
\delta\left(x_{0}, v^{\prime}\right)+\delta\left(v^{\prime}, w\right) \geq \delta\left(x_{0}, c a\right)+\delta\left(v^{\prime}, w\right) \geq \delta\left(x_{0}, b c\right)+\varepsilon+\delta(v, w)
$$

and a contradiction is obtained as above.

Now, we have

$$
\delta\left(b, b^{\prime} c^{\prime}\right)-\delta\left(b, a^{\prime} b^{\prime}\right)=\delta\left(b, b^{\prime}\right)(\sin \gamma-\sin \alpha) .
$$

By choosing $q$ large enough, the above difference can be made as large as wished; we make it larger than $\varepsilon$. Suppose now a (rectifiable) path from $x_{0}$ to $b^{\prime} c^{\prime}$ crosses $a b c$ at $y$. Then its length is at least

$$
\begin{aligned}
\delta\left(x_{0}, y\right)+\delta\left(y, b^{\prime} c^{\prime}\right) & \geq \delta\left(x_{0}, u\right)+\delta\left(b, b^{\prime} c^{\prime}\right) \geq \delta\left(x_{0}, u\right)+\delta\left(b, a^{\prime} b^{\prime}\right)+\varepsilon \\
& >\delta\left(x_{0}, u\right)+\delta\left(c, a^{\prime} b^{\prime}\right) \geq \delta\left(x_{0}, u\right)+\delta\left(u, a^{\prime} b^{\prime}\right) \geq \delta\left(x_{0}, a^{\prime} b^{\prime}\right),
\end{aligned}
$$

so it cannot be a shortest path from $x_{0}$ to $a^{\prime} b^{\prime} c^{\prime}$. Analogously, no path from $x_{0}$ to $c^{\prime} a^{\prime}$ is a shortest path from $x_{0}$ to $a^{\prime} b^{\prime} c^{\prime}$. This completes the proof of all desired properties for $P$. It is clear that, iterating this procedure, the shortest path from $x_{0}$ to the last constructed triangle has a steadily increasing spiralling number (by a rate close to $\frac{1}{3}$ for each new triangle). Thus $s(P)$ can be made as large as we wish. The theorem is proved.

Remark 3. In the above construction the path $P$ can be forced to turn, each time, right or left, as wished.

In [5], Pach gives a neat example of a polytope $K \subset \mathbb{R}^{3}$ and a shortest path $P$ on the boundary of $K$ with non-smooth points $z_{0}, \ldots, z_{n}$ such that $\sum_{i=1}^{n} \gamma_{i}$ is not bounded. Here $\gamma_{i}$ is the angle between the outer normals of the facets containing the segments $\left[z_{i-1}, z_{i}\right]$ and $\left[z_{i}, z_{i+1}\right]$. We mention that our construction has the same property. 


\section{References}

1. P. Agarwal, S. Har-Peled, M. Sharir, and K. Varadarajan, Approximating shortest paths on a convex polytope in three dimensions, J. A. C. Math. 44 (1997), 557-584.

2. K. Borsuk, Sur la courbure totale des courbes fermées, Ann. Soc. Polonaise 20 (1947), 251-265.

3. W. Fenchel, Über Krümmung und Windung geschlossener Raumkurven, Math. Ann. 101 (1929), 228-252.

4. J. W. Milnor, On the total curvature of knots, Ann. of Math. 52 (1950), 248-257.

5. J. Pach, Folding and turning along geodesics in a convex surface, Geombinatorics 7 (1997) 61-65.

6. A. V. Pogorelov, Extrinsic Geometry of Convex Surfaces. Translations of Mathematical Monographs 35, American Mathematical Society, Providence, RI, 1973.

Received August 17, 2001. Online publication July 10, 2003. 